Language and Cognition 7 (2015), 1-35. doi:10.1017/langcog.2014.15

(C) UK Cognitive Linguistics Association, 2014

\title{
A principled Cognitive Linguistics account of English phrasal verbs with up and out*
}

\author{
NARGES MAHPEYKAR \\ AND \\ ANDREA TYLER \\ Georgetown University \\ (Received 16 September 2013 - Accepted 02 February 2014 - \\ First published online 16 May 2014)
}

\section{A B S T R A C T}

Many attempts have been made to discover some systematicity in the semantics of phrasal verbs. However, most research has investigated the semantics of particles exclusively; no study has examined how the multiple meanings of the verb also contribute to the meanings of phrasal verbs. The current corpus-based (COCA) study advances the research on phrasal verbs by examining the interaction of the polysemy networks of both the verb and the particle in four phrasal verb constructions: get up, take up, get out, and take out. Following the Cognitive Linguistics (CL) based methodology set out by Tyler and Evans (2003) for analyzing the semantics of particles, in conjunction with Langacker's (1991) analysis of the semantics of verbs, a replicable polysemy analysis of the semantics of get and take was established. The polysemy networks for both the verbs and the particles laid the foundation for investigating the multiple meanings of the phrasal verbs found in the corpus. The CL-based analysis of the semantics of the phrasal verb constructions provides evidence for the compositional nature of phrasal verbs, showing that the multiple meanings can be systematically accounted for through the interaction of the polysemy networks of the component verbs and particles.

KEYWORDS : conceptualization, compositionality, embodiment, phrasal verbs, polysemy, semantic network.

[*] Address for correspondence: Narges Mahpeykar, Department of Linguistics, Georgetown University. e-mail: nm352@georgetown.edu 


\section{Introduction}

The semantics of English phrasal verbs and their multiple meanings are a well-recognized challenge in English. In spite of several attempts at analysis (e.g., Bolinger, 1971; Kennedy, 1920; Live, 1965), the multiple meanings of these lexical units have been extensively regarded as arbitrary and unpredictable (Celce-Murcia \& Larsen-Freeman, 1999; Fraser, 1976; Lipka, 1972). Traditional approaches have, in general, failed to provide a systematic, consistent account for the semantic behavior of phrasal verbs. However, recent advances in Cognitive Linguistics (CL) have begun to provide some evidence for systematicity of these constructions, primarily through examining regularities found in the polysemy of prepositions and particles (Brugman, 1981; Brugman \& Lakoff, 1988; Lakoff, 1987; Lindner, 1981; Rudzka-Ostyn, 2003; Taylor, 2002; Tyler \& Evans, 2001, 2003). Despite the insightful research into the polysemy of spatial particles, CL studies have not yet provided a detailed, rigorous explanation of the semantics of phrasal verbs. We argue that much of the lack of progress is due to the fact that the semantics of phrasal verbs has only been analyzed in terms of the meanings of the particle, without consideration of the polysemy networks of the verb with which the particle is combining. This study advances the research on the semantics of phrasal verbs by examining the interaction between the polysemy networks of the verb and the particle in a set of high-frequency phrasal verbs including get up, take up, get out, and take out. The study provides a detailed account of the complex semantic networks of these phrasal verbs, showing that the multiple meanings are compositionally formed through the interaction between the polysemy networks of both the verb and the particle.

$\mathrm{CL}$ research has shown that word polysemy is motivated, suggesting that the multiple meanings of phrasal verbs should also exhibit some degree of systematicity (e.g., Brugman \& Lakoff, 1988; Deane, 2005; Dirven, 2001; Lakoff, 1987; Lindner, 1981; Newman, 1996; Rudzka-Ostyn, 2003; Taylor, 2002; Tyler \& Evans, 2001, 2003; Vandeloise, 1991). A primary concern of $\mathrm{CL}$ research has been with the semantics of the particles and their polysemous nature (also see Evans', 2006, 2009, Theory of Lexical Concepts and Cognitive Models or LCCM for analysis of nouns, and Newman, 1996, for analysis of give). According to CL studies, many of the multiple meanings associated with prepositions and particles are related in organized ways, forming systematic polysemy networks (e.g., Lakoff, 1987; Littlemore \& Low, 2006; Taylor, 2002; Tyler \& Evans, 2001, 2003). The cognitive semantic frameworks proposed by these studies have produced useful models for accounting for much of this polysemy in terms of the radial categories and semantic networks extending from a central or prototypical sense. The underlying principle central to all these studies is that particles constitute complex categories, 
which are conceptual in nature, and in order to determine their complex array of meanings it is necessary to identify the cognitive mechanisms employed by humans in real language use and meaning extension.

We hypothesize that understanding the compositional nature of phrasal verbs can shed light on the semantics of these lexical units, including the multiple meanings they exhibit. Moreover, identifying the various meanings of verbs and particles can provide insight into what motivates new uses of a phrasal verb. More specifically, this study aims to achieve the following goals:

1. To provide a CL analysis of a set of highly frequent and polysemous English phrasal verbs by applying Tyler and Evans' (2003) Principled Polysemy Model, in conjunction with Langacker's $(1987,1991)$ analysis of the semantics of verbs.

2. To investigate the ways in which the semantic networks of the verb and particle in phrasal verb constructions interact with each other, and to determine the degree to which their meanings are compositional.

The phrasal verbs selected for the analysis consist of a set of frequent and highly polysemous English verb-particle combinations. The particles examined in this study are $u p$ and out, which combine with the polysemous verbs get and take. The semantic analyses of $u p$ and out are based on Tyler and Evans' (2003) proposed polysemy network of these spatial particles; the semantic networks for get and take were developed specifically for the present study and drew largely on the methodology established by Tyler and Evans for the analysis of spatial particles, in conjunction with Langacker's (1987, 1991) analysis of the semantics of verbs. The analysis of these phrasal verbs is based on a set of naturally occurring data randomly extracted from the Corpus of Contemporary American English (COCA). ${ }^{1}$ The data include 200 instances of each verb-particle combination (800 total tokens). The semantic analysis involves identifying the central sense and the extended senses for each phrasal verb, providing a precise account of the various meanings associated with these verb-particle combinations.

\section{Literature review}

2.1. CL APPROACHES TO THE SEMANTICS OF PHRASAL VERBS Lindner (1981) provides a detailed cognitive semantic analysis of the particles $u p$ and out. The central claim of this study is that particles almost invariably contribute to the meaning of verb-particle constructions (VPCs). In Lindner's

[1] <http://corpus.byu.edu/coca $>$. 
view, previous approaches, including Fraser (1976), incorrectly claimed that particles do not have meaning, and the majority of phrasal verbs are listed in the lexicon as arbitrary forms. Challenging this view, she provides a detailed CL analysis of the two particles, suggesting that these lexical items have both concrete and abstract meanings, with each set of meanings participating in a single, unified, semantic network. This analysis assumes a central super schema for each particle from which the rest of the senses are extended in an organized way. Despite Lindner's insightful analysis, her study has a number of gaps. One is related to the semantics of the verb. While Lindner discusses some aspects of the verb's syntactic properties participating in VPC constructions, not much attention was dedicated to the semantics of the verbs and their polysemous nature. In addition, the methodology for identifying the central sense and the various senses of $u p$ and out is not fully explained in this study, rendering it challenging for a replicable analysis of the particles and the phrasal verb constructions in which they participate.

In a more recent study, Rudza-Ostyn (2003) applies a CL analysis of verbparticle constructions to course materials. In order to present the CL analysis of phrasal verbs, she offers her own view of plausible motivations for a large number of phrasal verbs. In this approach, the different meanings of the particles are categorized based on a central image-schema involving a specific relationship between trajector (TR) and landmark (LM); the analysis allows for a diagrammatic representation of each particle's spatial meaning. In order to motivate many of the extended meanings of English particles, RudzkaOstyn draws on the theory of conceptual metaphor proposed by Lakoff (1987). For instance, according to Rudzka-Ostyn, the metaphor H U M A N BODY/MIND IS A CONTAINER motivates the use of a number of phrasal verbs including stuck out and thought out in the following sentences:

i. The little girl stuck out her tongue.

ii. She was so clever, she always thought out a solution to the problem.

In sentence (i) the mouth of the little girl is conceptualized as a container from which the tongue is moving from inside the mouth to the outside region, and in sentence (ii) the mind is conceptualized as a container from which thoughts emerge (a metaphor for finding a solution to a problem). ${ }^{2}$

Despite offering some systematicity for the multiple meanings of phrasal verbs, a number of shortcomings have been expressed concerning

[2] In Tyler and Evans' (2003) account the sense of out participating in (i) is the Reflexive Sense and in (ii) is the Knowing Sense (more detail on the senses of out identified by Tyler and Evans will be provided in Section 4.3.1). 
Rudzka-Ostyn's approach. One major problem is the failure to provide a methodology for identifying the central sense of the particle. Nor does she account in any systematic way for how the different meanings are extended from this basic sense. In addition, the reasoning used to determine some of the senses is not explained, and not all the examples seem to accurately represent a designated sense. Finally, her analysis focuses solely on the semantic contribution of the particles.

The study by Andrea Tyler and Vyvyan Evans (2003) provides a carefully articulated set of linguistic tools with which to analyze lexical polysemy, in particular the polysemy of prepositions. Motivated by the goals of providing a replicable methodology, the researchers developed a framework of semantic analysis referred to as the Principled Polysemy Model. The model aims to provide (i) a replicable method for identifying the central or basic sense from which the extended senses are derived systematically, and (ii) a set of criteria for determining whether a specific use constitutes a distinct sense of a particle. These principles are important because they address methodological issues that have not been previously accounted for by other CL approaches, such as Brugman and Lakoff (1988) and Lakoff (1987).

In this approach, the analysis of prepositions relies on recognized principles of cognition such as knowledge of force dynamics and embodied experience (Johnson, 1987; Lakoff \& Johnson, 1980; Mandler, 1992; Sweetser, 1990; Vandeloise, 1991). Tyler and Evans (2003) argue that language directly refers to the human conceptual system rather than the 'real world'. The conceptual structure is largely mediated by how we as humans experience and interact with objects and the spatial-physical world around us; in other words, experience is embodied. Consequently, many of the meanings of prepositions are derived from our embodied experience and conceptualization of the spatial-physical world. For instance, our frequent daily interaction with containers and containment has given rise to multiple uses of in and out. The model relies on cognitive processing, including pragmatic inferencing and experiential correlation (Grady, 1997, 1999; Hopper \& Traugott, 1993; Johnson, 1999; Lakoff, 1987; Langacker, 1987; Talmy, 2000; Traugott, 1989). Experiential correlation together with pragmatic inferencing implies that the frequent correlation between two events in everyday experience can lead to the association of a new meaning with a particular lexical form. The association occurs through the continued use of the form in particular contexts in which the implicature occurs. For instance, in our daily experience we encounter a recurring correlation between quantity and vertical elevation, such as when objects are added to a pile or liquid is added to a container. In these instances an increase in quantity correlates with an increase in vertical elevation. The ubiquitous observation of these two co-occurring phenomena results in a strong cognitive association between the two. This conceptual association, 
in turn, is reflected in language such as The prices have gone up, in which language from the domain of vertical elevation is used to talk about an increase in amount (e.g., Grady, 1997; Johnson, 1999; Lakoff, 1987).

A key challenge for the precise analysis of all polysemy networks is to identify the central or core meaning. The central sense is the one from which the rest of the meanings are extended systematically. These criteria provide a significant body of evidence for systematic, replicable means of identifying the central sense. Tyler and Evans (2003) propose a number of criteria for identifying the central sense, which are listed below:

1. considering the etymological roots, including the earliest attested meaning;

2. taking into account the predominance of the sense in the polysemy network;

3. comparing the spatial relations to other prepositions

In addition to the above criteria, Tyler and Evans (2003) emphasize that the central meaning of each preposition involves the spatial configuration between a TR and a LM, as well as a functional element. The functional element is an important part of the central sense, which represents the humanly salient consequences of the TR and LM being in that specific spatial configuration. For instance, Tyler and Evans (p. 67) posit the functional element of over to be the relation of 'proximity' or one in which "the TR and LM are in each other's sphere of influence”. A consequence of such relation between the two elements is that in certain construals the TR which is the higher entity in this relation can possess power and control over the LM. Tyler and Evans' Principled Polysemy Model will be used in the current study.

\subsection{COMPOS I T I ONALITY}

Many traditional approaches, including formal semantics, suggest a strictly modular view of language (Gamut, 1991; Montague, 1974; Quine, 1970). In formal semantics, meaning is taken to be mathematically based and rule-governed, analogous to traditional representations of syntax as rulegoverned. Lexical items are viewed as 'building blocks' that are lined up in different arrangements (syntax) to form complex expressions, with meaning being constructed out of the aggregate of the individual linguistic components. Each lexical item contributes a portion of literal meaning; no additional meaning, beyond the compilation of meanings contributed by the individual lexical items, accrues. In formal semantics this is known as 
the FULL COMPOSITIONALITY approach. Importantly, it assumes a clear distinction between semantics and pragmatics. Under this view, the core meaning of a sentence is its truth-conditional value, i.e., the conditions under which the sentence holds true. Truth conditions are equated with the propositional content, which is inherently independent from the context. For instance, the sentence $I t$ is raining outside is true if and only if it is raining outside, regardless of the intention of the speaker. According to this approach, meaning can be directly derived from the composite structure itself; any additional facets of meaning attributed to the utterance are explained by pragmatics (Cann, 1993; Langacker, 1987, 2008; Taylor, 2002).

Unlike the traditional approaches, cognitive semantics claims that semantics and pragmatics form a continuum, with no strict boundary between the two. Instead of being fully predictable, the meaning of lexical items is based on domains of knowledge which are activated by the communicative context and the interlocutors' background knowledge. According to this approach, meaning is conceptual in nature and the ways of CONSTRUING the content are an important part of the meaning. The cognitive semantics approach does not deny the existence of the compositional patterns, but considers them insufficient to explain the multiple, complex interpretations regularly assigned to utterances. The meaning produced from semantic composition of the lexical items and the sentence-level constructions in which they occur provides only some of the resources used in the process of creating and interpreting complex expressions. Other sources include our knowledge of the world, the meaning provided by the context, and cognitive mechanisms such as metaphor, metonymy, and mental spaces. In the cognitive semantics tradition, language is only PARTIALLY COMPOSITIONAL rather than fully compositional (Evans \& Green, 2006; Goldberg, 1995; Langacker, 1987, 2008; Taylor, 2002).

Langacker's approach to partial compositionality provides a useful framework for understanding the semantics of composite structures. In analyzing the meaning of symbolic structures, Langacker (1987, p. 277) speaks of "a grammatical valence relation" between the component structures. Valence relations are the ways in which the internal structures of two components interact to subsume a new linguistic meaning. The relations consist of four major factors, two of which are related to the analysis presented in this study: (i) correspondence and (ii) profile determinacy. According to Langacker (p. 278) CORRESPONDENCE can be regarded as an "overlap between two conceptions that permits their integration to form a coherent scene"; they can also be viewed as "instructions for assembling a composite structure from its components" (p. 278). PROFILE DETERMINACY refers to "the extent that the profile of a component structure is adopted as the profile of the composite structure" (p. 288). 
In this view, different aspects of the component entities are conceptually integrated in a way that their internal properties merge. Through merging, the profile of one of the component structures is adopted as the dominant profile of the scene. For instance, in the expression over the bridge, the two components over and the bridge integrate due to their overlapping substructures, and the profile of over becomes the dominant profile. Composite structures can be formed from any two component types, including those of relational status. An example of this is the English past participle, such as gone, which inherits two relational components go and PRTC (past participle). The composite structure which is formed has certain characteristics: (i) it is "experientially distinct from recognition of the individual components"; and (ii) "it may involve entities and specifications beyond those provided by the components" (Langacker, 1987 , p. 281). Identifying these correspondences is required for the full description of a construction since they provide information on the nature and degree of compositionality.

A similar type of analysis can be applied to phrasal verbs. Frequently used phrasal verbs such as get $u p$ are conventionalized and inherit a unit status in the proficient speaker's mind, and need only to be activated, yet their composite structure can be explained by examining the component structures. For instance, the semantic components of get up are: get (predicate 1) and $u p$ (predicate 2). In this construal get profiles a dynamic relation, i.e., one which unfolds through time, while up profiles a static relation between the spatial elements: trajector and landmark; both are in the domain of space. The integration of the two predicates depends on the correspondence between the process profiled by get and the schematic relational status of $\mathrm{TR}$ and $\mathrm{LM}$ serving as the base for the particle. By merging the specifications of these corresponding entities and adopting the profile of get, we obtain the composite structure which designates the dynamic relation constituting the process of get and the spatial configuration denoted by $u p$. In this relation, since the overall meaning of the phrasal verb designates a dynamic relation or process which unfolds through time, get is the profile determinant of the construction. The overall correspondence between the two relational predictions must be understood as subsuming a series of more detailed correspondences including that of their TRs, their LMs, and the relational status of the TR and LM. This is consistent with the designation of a verb as denoting a process which takes place over time; each stage of the process represents a particular correspondence between the TR and LM. While in some constructions, such as over the bridge, the nominal serves as the participant for the preposition, in phrasal verb constructions the verb and the particle are both relational and neither is considered the participant of the other. 


\section{Methodology}

In this study, four phrasal verb constructions which share the same two verbs and the same two particles are analyzed. The phrasal verb constructions are get up, get out, take up, and take out. These verbs were selected because, based on an examination of the entries in the online dictionaries WordNet $^{3}$ and the Cambridge Learner's Dictionary, ${ }^{4}$ as well as the COBUILD Dictionary of Phrasal Verbs, ${ }^{5}$ we determined that the verbs get and take were highly polysemous, high frequency, and combined with many particles in phrasal verb constructions. Following Tyler and Evans (2003), we verified that $u p$ and out are highly polysemous particles. An examination of the COBUILD Dictionary of Phrasal Verbs showed that these particles participated in numerous phrasal verb constructions, including several involving get and take. Additionally, the Corpus of Contemporary American English (COCA) ${ }^{6}$ lists them as the two most frequently occurring particles.

COCA was used for investigating the different uses of the phrasal verbs in naturally occurring data. The corpus consists of a variety of genres including spoken (unscripted conversation) from TV and radio programs, fiction, magazines, news, and academic journals. Two hundred instances of each phrasal verb were randomly extracted for a total of 800 tokens.

The coding scheme involved a recursive process, which is slightly different for the verbs and the particles.

For the verbs, the first step in the analysis involved identifying the central or core meaning. For this purpose, we began by establishing the etymological roots of the verbs based on the information provided in the Online Etymology Dictionary $^{7}$ in combination with COCA data. For instance, the earliest attested meaning of get provided in this dictionary is geta 'obtain'. If the original meaning is still currently in use and frequent, we posited it as the central meaning (for instance, this was the case for get for which the 'obtain' sense constituted 56 of 200 randomly sampled uses). Next, we consulted online dictionaries such as WordNet and the Cambridge Learner's Dictionary for potential multiple, extended meanings. According to these sources, take has 41 attested uses and get has 37 . However, close analysis of the sentential contexts revealed that many of these 'distinct' senses are contextual variants, with much of their purported 'distinctness' arising from context. This situation is analogous to the overly fine-grained polysemy analyses of prepositions

[3] WordNet: <http://wordnet.princeton.edu/> (last accessed July 2013).

[4] Cambridge Learner's Dictionary (2011). New York: Cambridge University Press.

[5] Collins COBUILD Dictionary of Phrasal Verbs (1994). Edited by John Sinclair and others. London: HarperCollins Publishers.

[6] COCA: <http://corpus.byu.edu/coca/> (last accessed July 2013).

[7] <http://www.etymonline.com>. 
discussed by Tyler and Evans (2001, 2003). In our analysis of the verbs, we drew on the established CL principle that much of the meaning of lexical items which represent actions and events are best understood as prompting for spatial scenes rather than sets of semantic features or propositional content (e.g., Tyler, 2008, 2012). Following Tyler and Evans, we adopted the criterion that a distinct sense must contain additional meaning not apparent in other proposed senses. In contrast to a central sense for a verb, an additional, extended sense: (i) need not be strictly physical/spatial in meaning; or (ii) the extended sense prompts for a different spatial scene. For instance, one clearly established sense for take is 'remove an entity from its original location', as in Alice took the letter from the desk and dropped it in her pocket. We argue that this meaning involves a spatial scene in which an actor grasps the letter and moves it from its original location. A second sense involves the notion of 'ingesting', as in It's time to take your medicine. Here the interpretation of take goes beyond simply moving a medicine from its original location; rather, the interpretation focuses on the placing of the medicine in the patient's mouth and the patient swallowing it. Using this methodology, we determined that the dictionary meanings for both verbs could be consolidated into substantially fewer categories (7 major meaning categories for take and 5 major meaning categories for get). These analyses further revealed that for each of the verbs one broadly defined sense predominated: for take the sense of 'grasping something and removing it from its initial location' was very closely related to 14 of the dictionary uses, and for get the meaning 'obtain/acquire' was very closely related to 21 of the uses.

As we identified the distinct senses, we developed our conceptualization of the central sense. As we mentioned above, we were guided by the hypothesis that the earliest attested meanings would be a potential central sense. A second important tenet was that the extended meanings could be traced back to the central sense in straightforward ways using established CL principles for meaning extension, such as experiential correlation and shifting construals on a scene. Considering the earliest attested meaning for both verbs showed that the early meanings were closely related to the predominate senses. Hypothesizing that these predominate meanings were the central senses for each verb, the remaining consolidated uses were analyzed to determine if these senses could be straightforwardly related to the hypothesized central sense. The analysis revealed that all but a handful of uses could be related to the proposed central senses.

For analyzing $u p$ and out, we initially applied the polysemy network of these particles proposed by Tyler and Evans (2003). The proposed semantic networks for these particles were refined in the course of analyzing the phrasal verb meanings in the data. For instance, the data revealed an Activity Sense for $u p$ which was not discussed by Tyler and Evans. 
In identifying the different meanings for the phrasal verb constructions we relied heavily on the COBUILD Dictionary of Phrasal Verbs, as well as Merriam-Webster online dictionary. ${ }^{8}$ After a preliminary analysis, we re-evaluated and consolidated the categories from the COBUILD dictionary, analogous to the process used for establishing the central and extended senses of get and take. Next, we formulated a coding rubric for the central and distinct senses of each targeted phrasal verb construction and applied it to the COCA data. The final refinement of the coding rubric involved a recursive process, as during the course of identifying the distinct uses of the phrasal verbs in the corpus, we were continually guided by the distinct contributions of the verb meanings and the particle meanings. As mentioned above, in the course of this phase of the analysis, we discovered uses of the particles which had not been part of the initially posited polysemy networks. These new uses were added to the rubric as the data demanded. Once the rubric was refined, two native speakers of English independently coded $20 \%$ of the data (40 tokens for each phrasal verb), yielding an inter-rater reliability percentage of $95 \%$ agreement.

\section{Analysis}

This section provides a detailed analysis of the selected phrasal verbs based on the corpus investigation. First, a brief description of the core meanings of get and take is provided. Following this, the semantics of each of the particles $(u p, o u t)$ and the phrasal verbs produced from the combination of each verb with the two particles is provided.

4.1. SUMMARY OF VERB MEANINGS

\subsubsection{Get}

The verb get is polysemous, in addition to being highly frequent. As we noted above, the online dictionaries list up to 37 meanings for get and our CL-based analysis established a central sense and 4 distinct extended clusters of senses. However, the senses of get which participate in instances of phrasal verbs found in the COCA corpus were limited to only the central sense and two distinct meanings. ${ }^{9}$ These extended meanings include the Move Sense, where the TR moves or is moved from one location to another (I got there on time), and the Change of State Sense, where the TR undergoes

[8] Merriam Webster: <http://www.merriam-webster.com/> (last accessed March 2013).

[9] We would like to thank Kaley Alberty for her assistance in identifying the semantics of the verbs in this study. 
an experience or change in condition ( I got hungry, I got cold, I got sick). Our analysis suggests that both these meanings are extensions from the Obtain Sense, which strongly relates to the meaning of to obtain from the Old Norse word geta (Online Etymology Dictionary ${ }^{10}$ ) and which we take to represent the central or core sense of the verb get. Figure 1 represents the central sense of get. Following Langacker, the meaning of the verb get is represented by a series of relationships between the TR and LM, which change over time. At stage 1, the hand is moving toward the object, but not yet in contact with it; at the final stage, a hand is wrapped around the object (TR) and thus in possession of the object. The time element is shown by an arrow. The Obtain Sense highlights the final TR-LM configuration in which the human participant has successfully grasped the targeted object.

Sentences from the corpus that illustrate this central sense include:

\section{(1) I got a book / I got a present}

Note that the Obtain Sense of get is ambiguous in terms of the agency of the 'obtainee'. In both these sentences the participant in subject position could either be an agent who actively obtained a book, or a recipient. Consider the following scenarios: I went to the library and I got a book about hummingbirds or A: What have you been doing all afternoon? B: I went to the department store and got a present (for my friend). In these scenarios, the common interpretation is that the participant acted as an agent who intentionally obtained a book or a present. Now consider the sentences in (1) from the perspective of a child opening a present given to her by someone else. From this perspective, the participant in subject position in I got a book or I got a present is in the non-agentive role of recipient. This ambiguity is manifested in some of the different senses listed in the dictionaries. For instance:

(2) a. What did you get at the toy store?

b. She got a lot of paintings from her uncle.

Sentence (2a) illustrates the obtainer in the agent role; sentence (2b) illustrates the obtainee as undergoer or receiver variant. The dictionaries listed these as two distinct senses; we consolidated them into one broad meaning related to obtaining or acquiring. We hypothesize that this same ambiguity will manifest itself in certain phrasal verb constructions.

[10] Online Etymology Dictionary: <http://www.etymonline.com/> (last accessed March 2013). 


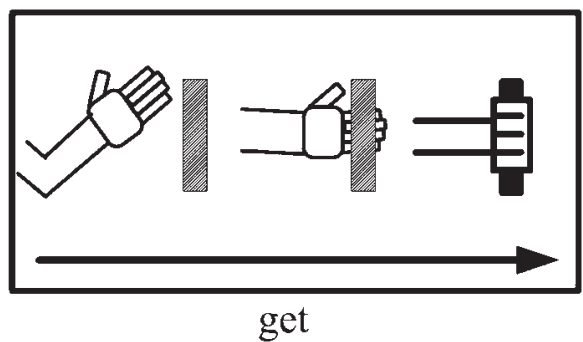

Fig. 1. Central sense of get. The TR is represented by a rectangle and the time element is represented by an arrow. The final TR-LM configuration is bolded, indicating that it is highlighted in the scene.

Embodied experience sheds light on how the core Obtain Sense became extended to the Move Sense. In order for humans to get, or obtain, an object, they most typically must move part or all of their body. For instance, in order to get (obtain) food we might have to move our entire selves to the kitchen and obtain what we want by moving our arms and our hands. Frequent experiences such as this, which necessitate moving our body (or part of it) in order to obtain objects, have given rise to the Move Sense of get. This meaning of get highlights the first two TR-LM configurations in Figure 1 in which the arm changes position in order for the hand to become close enough to the TR object to grasp it.

The Change of State Sense of get also extends from the Obtain Sense. Notice that the diagram in Figure 1 clearly involves the hand and arm undergoing a change of location. This requisite change of location inherent in the meaning of get gives rise to the Change of State Sense. A pervasive human experience is that as we move from one location to another, we experience changes in the environment. Often these changes in the environment are accompanied by a change in our physical or emotional states. For instance, if we change locations by walking from the bottom of a hill to the top of a hill, we are likely to experience a sense of physical exertion and loss of energy. Or, if we are initially in a vulnerable physical location, for instance outside in the rain, and we move to a location of greater protection, as in a building which gives us shelter from the rain, we experience not only a change of location, but a change of physical and emotional comfort.

The embodied experience of physical change of location correlating with change of state is reflected in three pervasive conceptual metaphors in English: (i) MENTAL AND PHYSICAL STATES ARE OBJECTS; (ii) (MENTAL AND EMOTIONAL) STATES ARE LOCATIONS; and (iii) CHANGE OF LOCATION IS CHANGE OF STATE. The combination of these three metaphors are illustrated in sentences such as: 
(3) I caught a cold. / The students gave me the flu. (STATES ARE OBJECTS; CHANGE OF LOCATION IS CHANGE OF STATE)

(4) He captured his family's loyalty. / I'll send all my loving to you. (STATES ARE OBJECTS; STATES ARE LOCATIONS)

When a person goes from a state of being healthy to a state of being unhealthy (for instance, either with a cold or the flu), the viruses in the environment penetrate their body and thus come to reside within the body. The viruses have changed location. As a result of contracting these harmful elements, the person's physical state is changed. Even before knowledge of viruses, bacteria, and cells, humans conceptualized going from a healthy state to an unhealthy state as somehow being caused by the person coming into possession of or being invaded by a 'bad' element.

The following examples provide minimal pairs which illustrate the three different meanings of get: the core Obtain Sense and the two extended senses, the Move Sense and the Change of State Sense.

(5) I got a book / I got a present (core Obtain Sense)

(6) I got across the bridge / I got to school on time (Move Sense)

(7) I got hungry / I got upset when I heard the news (Change of State Sense)

\subsubsection{Take}

The WordNet dictionary lists 41 distinct meanings for this verb. As discussed above, our CL-based analysis of take established a central sense and 6 distinct extended sense clusters for this verb. However, the senses of take participating in instances of phrasal verbs found in the COCA corpus and examined in this study only exhibit the central sense and one extended meaning. Take's etymological origin is from Old English tacan, which meant 'grasp' or 'lay hold of' (Online Etymology Dictionary). After recursive coding of the data (see above), it was determined that the central sense of take is what we term the Get Hold Of and Remove Sense. Figure 2 represents the central sense of take. The meaning of take is depicted by a series of scenes in which a hand is initially moving toward an object, then the hand grasps the object, and subsequently the hand and arm move the object toward the actor (as indicated by the final bent elbow in the diagram). Thus, the central sense of take involves movement of the TR (object), as well as movement of the actor's hand. There seems to be an important element of intention to remove the object from its original location which is highlighted when we contrast take and give. We find a key difference in the deictic center - give highlights a possessor 


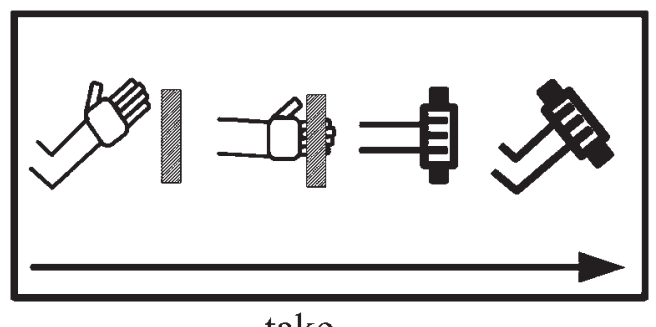

take

Fig. 2. Central sense of take. The TR is represented by a rectangle and the time element is represented by an arrow. The final two TR-LM configurations are bolded, indicating that these aspects of the scene are highlighted.

causing an object to be transferred to a receiver; take highlights an intentional agent becoming a possessor. Notice that the action depicted in the Get Hold Of and Remove Sense is punctual in nature, as in the sentence The squirrel took the nut. The final two TR-LM configurations, grasping the object and removing it from its initial position, are highlighted in the central sense of take.

In addition to this central meaning, we identified one extended sense for this verb in the corpus, the Extended Use or Occupy Sense. A consequence of grasping an object is that the agent becomes collocated with it. Moreover, a natural consequence of a person obtaining an object is that they will use the object. Importantly, not all instances of an agent being collocated and using an object involve physically grasping the object. For instance, in the situation of an agent sitting in a chair or seat, the TR (agent) is collocated with and using the LM (chair/seat), without the entailment that the agent is physically grasping the chair. For example, if a person takes a table at the library, we conceptualize the spatial scene as the TR (agent) sitting at or using the LM (the table), not as the agent physically grasping the table and potentially moving it to a different location. Frequent observation of this type of collocation between an agent and a LM has given rise to the meaning of Extended Use (EU) / Occupy for take. The EU/Occupy Sense has, in turn, been extended into the temporal domain. When the EU/Occupy Sense is used, occupying an object (e.g., taking a table or taking a seat) implies a durational event as opposed to a punctual event (e.g., taking a pen from the desk). This durational quality is an important part of the temporal extension in expressions such as take the time to do it right. The third TR-LM configuration, that of grasping the object, is highlighted in the EU/Occupy Sense.

The following examples provide minimal pairs which illustrate the two different meanings of take: the core Get Hold Of and Remove Sense and the EU/Occupy Sense. 
(8) I took a book / I took the job (core Get Hold Of and Remove Sense)

(9) I took my seat at the concert / It took time to write my dissertation (EU/ Occupy Sense and Temporal extension)

4.2. UP

This section presents the semantics of $u p$ and the phrasal verbs produced from the combination of the verbs get and take with this particle.

\subsubsection{Tyler and Evans' analysis of up}

According to Tyler and Evans (2003), the polysemy network of up is quite complex. This is because, as noted by Lakoff and Johnson (1980), there is a large set of embodied experiences and important consequences that correlate between a human being in a physically elevated position and how humans interact with surrounding entities. Tyler and Evans' discussion of $u p$ focuses on explaining the central sense and one cluster of senses: the Quantity cluster. For the most part, the analysis of the particle involves drawing on the asymmetrical nature of the human body and embodied experience. Humans have a top-bottom anatomical asymmetry due to the fact that we are biped and thus have a physical top-bottom asymmetry. Moreover, our primary organs of perception (vision, smell, taste, hearing) are located on our heads. This asymmetry gives rise to several meanings of $u p$. In addition, experiential correlations contribute to complex conceptualizations. The following section briefly presents the central sense and the extended meanings of $u p$ presented by Tyler and Evans.

The central sense of up. Tyler and Evans (2003, p. 136) posit that the central sense of $u p$ designates a relation in which "the TR is directed towards the top of an oriented LM". The LM is conceptualized as having a top part and a bottom part, and the TR is construed as being oriented. The meaning of $u p$ is formed by the relation between the oriented TR and the asymmetrical LM. Some examples of the central sense are provided below:

(10) The bird flew up the chimney.

(11) Jennifer climbed $u p$ the mountain.

Although in some cases the LMs are not necessarily asymmetrical, the asymmetry of the human body seems to project onto these structures. For instance, we can talk about the 'top' of the chimney or the 'peak' of the mountain. The human body offers a schema for the LM which is reflected in the central sense. Figure 3 shows the central sense of $u p$ suggested by Tyler and Evans (2003). 
In Figure 3, the 'stick' person represents the LM, which has a highlighted head or top that is in focus. The highlighted TR is represented by the dark sphere. The orientation of the TR is represented by the direction of the arrow rising from the sphere. The functional element or the interactive relation between the TR and LM posited for the scene is that of positive value. According to this analysis, there is a correlation between the actor being in a high position (or physically elevated) and the actor being in a state of readiness and increased control over the environment. In addition, there are a number of consequences for humans when other entities or objects are in a physically elevated position. For instance, when objects are raised to a vertically elevated position, they often become more visible or accessible or salient to the actor. This tight set of experiential correlations with $u p$ have given rise to a number of senses that were not explored in depth by Tyler and Evans (2003), but which surface in the phrasal verbs using $u p$.

The quantity cluster. The quantity cluster of senses primarily utilizes the previously discussed experiential correlation between quantity and vertical elevation, where an increase in quantity correlates with an increase in height. The concept of 'increase in amount' has become so entrenched in up's polysemy network that, through pragmatic strengthening, it has become an independent, established meaning which no longer necessarily refers to the original spatial scene. The extended meanings identified in this cluster are mostly non-spatial in nature, even though they are inherently grounded in spatial scenes related to the central sense. The following section explains the extended senses of $u p$ which are used in the analysis of phrasal verbs in this study.

More Sense. As previously mentioned, many times quantity and vertical elevation are conceptually related due to the frequent co-occurrence of these two phenomena. According to Tyler and Evans (2003), the association between the two gives rise to the More Sense of $u p$. The following examples illustrate this meaning of $u p$ :

(12) The maid plumped $u p$ the cushions.

(13) Turn $u p$ the volume.

In both examples, an increase in upward orientation correlates with greater amount. Although in (12) the context could possibly allow the listener to deduce the meaning, in (13) the implicature is not available from the context, which indicates that it is a distinct sense in the polysemy network.

Completion Sense. Another consequence of increasing in amount is that, in some cases, a limit or an endpoint is reached and the increase in quantity is complete. The most common example of this correlation is related to our repeated interaction with containers. For instance, if water is poured into a 


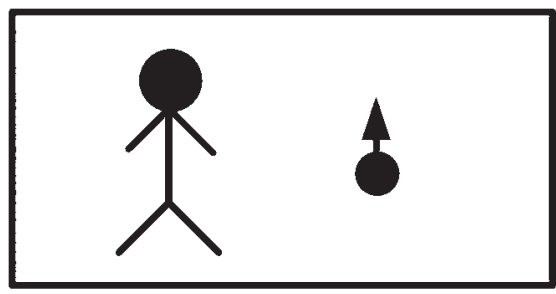

up

Fig. 3. The central sense of $u p$. The TR is represented by a dark sphere and the orientation of the TR is represented by the direction of the arrow emerging from the sphere. The stick figure represents the asymmetrical LM and the head or top is in focus and hence highlighted (Tyler \& Evans, 2003, p. 137).

cup, the vertical elevation of the water is increased until, at some point, the limit of the cup is reached. According to Tyler and Evans (2003), there is a correlation between the amount of liquid in the container increasing, an increase in vertical elevation (or in being up), and the capacity of the container being completely used, and in this way $u p$ has developed a Completion Sense. The following examples illustrate this sense of $u p$ :

(14) Be sure to gas $u p$ the car for the trip.

(15) Let's load $u p$ the truck and get going.

Through pragmatic strengthening, this implicature has given rise to a conventionalized meaning of completion. Interestingly, a different experiential correlation denotes a different aspect of the meaning of completion. The sense of completion here seems to involve the notion of depletion, which can be seen in the following examples:

(16) The flashlight won't work. We must have used $u p$ the battery.

(17) Turn in your papers, time is $u p$ !

In (16), up denotes that all the energy is consumed, and in (17), up denotes that the time allocated for testing is finished. According to Tyler and Evans (2003), the meaning of depletion derives from our basic experience of taking the drink to the mouth and consuming it, and through pragmatic strengthening the association between $u p$ and depletion has become conventionalized. Thus different kinds of experiential correlations can motivate meanings which are distinct but closely related.

The following section presents the analysis for the different verb-particle combinations with $u p$. For each phrasal verb, a central sense and a number of 
distinct meanings are identified. The analysis involves a detailed explanation of each sense, followed by a number of examples from the corpus.

\subsubsection{Analysis of get up}

Central sense of get up. In its central sense, the phrasal verb get up denotes a meaning of an entity moving (or being caused to move) from a lower position to a higher position. This meaning is compositionally formed through the combination of an extended sense of the verb and the central sense of the particle. In the central sense of get up, get denotes 'movement from point A to point B' and $u p$ highlights 'the relation between the oriented TR and the top part of the asymmetrical LM' ${ }^{11}$ Figure 4 shows the proposed central sense for get $u p$. The circle represents the TR and the rectangle represents the LM. Note that the full meaning of get is represented in three spatial scenes: (i) the TR is collocated with space A; (ii) the TR is located in between spaces A and B; and (ii) the TR is collated with space B. Here space B is interpreted as the actor who obtains the TR.

Two examples for the central sense of get up are provided below:

(18) If you're going to be a mountain climber, you've got to try and get up there.

(19) The astronaut says "Getting up and back is the hardest thing, and while you're up there it's not that benign either." (up there refers to outer space)

In addition to this basic meaning, some metaphorical extensions of this sense were observed in the corpus, including the following two sentences:

(20) I told him not to get his hopes $u p$, but it's worth giving it a shot.

(21) We got our guts up and went in the room.

In the above examples, hopes and guts (a metaphor for 'courage') are conceptualized as objects that can be elevated. This conceptualization makes it possible for us to talk about our emotions and feelings in terms of objects that can be lifted, and which can consequently be increased.

Distinct senses of get up. Analyzing the data using CL tools yielded three distinct senses for get $u p$.

[11] In some cases, such as in examples (18) and (19), get seems to have this additional meaning of struggling or overcoming a barrier. This is a natural consequence of overcoming the force of gravity in order to move from a lower position to a higher position. In (18), moving from the base of the mountain to the top is challenging, and in (19) getting beyond the earth's gravitational pull and into space seems to be a hard task for the astronaut. 


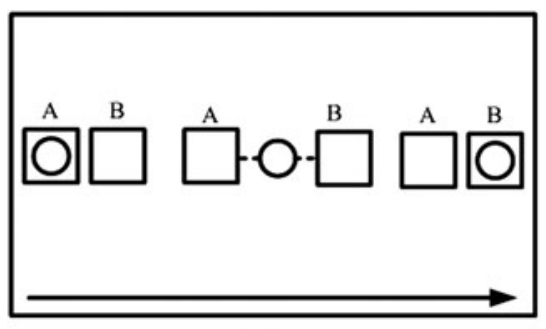

get

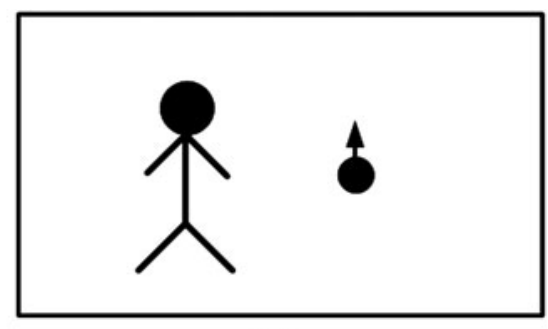

up

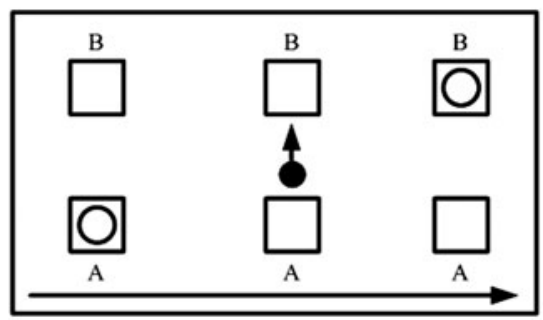

get up

Fig. 4. The central sense of get up. The time element is shown by an arrow and the bolded objects are profiled in the scene. In the spatial configuration for get and get up, the TR is represented by a circle, and State $\mathrm{A}$ and $\mathrm{B}$ are represented by individual squares.

Become upright. This sense denotes that the TR (an animate being) is rising to a standing position. This construction also draws on the Move Sense of get and the central sense of $u p$. The spatial configuration denoted by this scene is similar to the central sense of get up in that the TR is in position A at the beginning of the timescale, and is in position B at the end of the timescale. In distinction with the central sense, in the Become Upright Sense, at point A the TR (animate being) is necessarily sitting or lying down and at point B the $\mathrm{TR}$ is in a standing position. In other words, the key difference between this sense and the central sense is that in the central sense, the TR is not specified; in the extended sense the TR is oriented and the orientation of the TR itself changes from the horizontal to the vertical as the process unfolds. This meaning is directly tied to our embodied experience and the physical nature of our bodies. Some consequences of being in a position of sitting or lying down are that we are in less control of the surrounding environment and objects in our vicinity are often less visible. On the other hand, when we are standing up we tend to be more in control of our surroundings, are in a readiness position, and have more visual access to our surroundings. The following examples from the corpus illustrate the central sense of the verb: 
(22) I tried to get up, but he leaned over and forced me on my back.

(23) If a lady gets up from the table, you stand up, manners make a man.

Some metaphorical extensions of this sense were also observed in the data, which did not refer to the physical upright position of a human body. Instead, the use of get up in this case denoted a sense of being in control and in a superior position as opposed to being inferior, which is extensively tied to the embodied experience explained above. The following example in the corpus denotes this sense of get $u p$ :

(24) White people are always going to try to keep you down, and the only question you have to answer is if you're going to keep getting up.

Wake and move out of bed. This sense denotes both awakening and the physical movement of the body from a horizontal, resting position to an upright position. The complex semantics, and complex set of spatial scenes the phrasal verb evokes, suggests that get $u p$ involves multiple senses of both get and $u p$. The first stage in the process involves the Change of State Sense associated with get, as the actor/experiencer is moving from a state of being asleep or unconscious to a state of being conscious. The semantic contribution supplied by $u p$ is the Awake Sense that Lakoff and Johnson (1980) discuss as a basic experiential correlation associated with being in an $u p$ position. As they note, there are a wide range of physical experiences that humans associate with being upright. One of the most basic is being awake or conscious; after all, given gravitational forces and human anatomy, it is very difficult to remain upright and not be conscious. Notice that US English speakers can use $u p$ alone in a sentence to indicate that they are awake. Consider the following exchange:

(25) Mother Calling to TeEnager: Time to get up or you'll be late for soccer practice.

(26) TEENAGER: Ok, ok. I'm $u p$.

Here, I'm $u p$ is ambiguous and can be interpreted either as 'I'm awake' or 'I'm now out of bed'. This second stage denoted by get up, i.e., that the actor has moved from a resting position to an upright position, draws on get's Move Sense. The sense of $u p$ is the central sense of being in an upright position. This meaning is directly related to our embodied experience with the environment. In order to be out of bed, we move from a lying, horizontal position to an upright position. Due to the high frequency of this type of experience with our resting location (or bed), this sense has become a conventionalized, highly entrenched sense. Evidence for this being a distinct sense for get up comes from 
sentences such as I got up late, where the speaker's utterance is interpreted as specifically referring to awaking, then rising from the bed late in the day and not just the general meaning of Become Upright. Other examples in the corpus are:

(27) Every day we get up, wash, and eat soup.

(28) She would wake me up in a very special way. If she got me $u p$ that way now, I might think I'd died and gone to heaven.

Organize an event. In this construction, the meaning of get up is compositionally formed through the combination of the extended Change of State Sense of get and the extended Activity Sense of $u p$. As previously mentioned, a consequence of being $u p$ is that the TR/actor is in a state of readiness or active engagement. This distinct sense of $u p$ is illustrated in a sentence such as Hey, man, what're you up to?, which is commonly interpreted as 'What activities are you currently engaged in?' or the ubiquitous US English greeting: What's up?, which is commonly interpreted as 'What's going on?' or 'What activities are occurring?' The Change of State Sense here involves an initial state in which no particular event or activity has been planned or organized to a second state in which planning for such an event has taken place to the point that the event is about to begin. A consequence of having an object in an $u p$ position is that it is more accessible and more salient to the actor. In this use of get $u p$, events are conceptualized as objects that can be lifted from a lower position to a higher position, with an unplanned or unconceptualized activity having low saliency and a planned, about-to-happen activity having much higher saliency. The following examples illustrate this sense of get up:

(29) There were usually more people around at Christmas, so you could almost always get up a game.

(30) We are trying to get up an event for the Vineyard.

\subsubsection{Analysis of take up}

Central sense of take up. The central sense of take up denotes an entity in the hand which has been moved from a lower position to a higher position. The meaning of take in this combination is the central Get Hold Of and Remove Sense, and the meaning of $u p$ is the central sense of 'the TR being situated in a higher position'. In order to take an object $u p$, the actor's hand moves toward the object, and gets hold of it, followed by the actor moving the object (and hand) away from its original location to a vertically elevated position. As a consequence of holding the object, the person becomes collocated with it. For instance, a child can take a teddy bear and hold it; in this scenario, 
the child and the teddy bear are co-located. Figure 5 demonstrates the central sense of take up.

The spatial scene explained above is what we propose to be the central sense of take up. However, unlike the central sense identified for other phrasal verbs we have investigated, such as get $u p$, the central sense occurs relatively infrequently. Historically, there is evidence of the central sense found in the King James Bible. For instance, in the story of Elijah, he is exhorted to 'take up the mantle' (the cape of the prophet) which is lying on the ground. Examples found in the corpus include:

(31) The supporters on the street had taken up weapons, butchered their neighbors and made the country ungovernable.

(32) The boys went over and took up the wreaths and brought them towards the church, and the one in front wore roses around his neck like a horse that has won and can smile.

Distinct senses of take up. Two distinct, extended senses of take up were found in the COCA data, which are presented below.

Become engaged with an activity or an idea. This use involves the conceptualization of the actor becoming involved with an idea, issue, or activity. An integral part of becoming engaged with or involved with an idea or activity is that it becomes more salient to the actor/TR. The sense of take participating in this meaning is the central Get Hold Of Sense. A consequence of objects being in a vertically elevated position is that they are typically more visible and salient. Similarly, by taking an issue or activity $u p$, we are conceptualizing ideas or activities as objects that can be grasped and raised higher in the visual field and therefore be made more salient. Ideas and activities are also understood as entities with which the actor can be engaged. Thus, this extended meaning of take up crucially relies on the conceptual metaphor IDEAS ARE OBJECTS. The following examples from the corpus illustrate this sense of the phrasal verb:

(33) The question was later on taken up by the government.

(34) The 'Occupy' protesters took up the cause of financial reform of the banking system.

(35) I took up sports after I graduated.

In these examples, getting engaged with the issue or activity is considered something positive to the speaker, and by using take up (and not just take) the speaker is giving a sense of saliency to engagement with the idea or activity. 


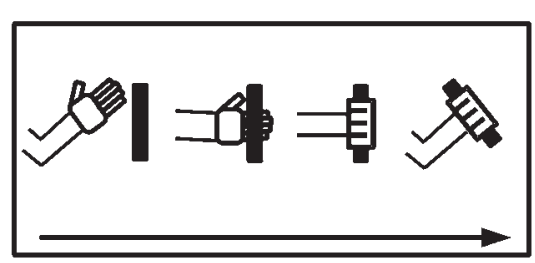

take

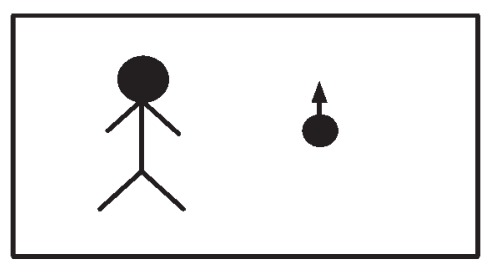

up

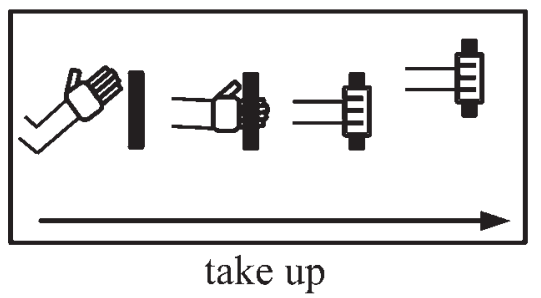

Fig. 5. Central sense of take up. The TR is represented by a rectangle. The time element is shown by an arrow and the bolded objects are profiled in the scene.

Occupy space or time. In the spatial scene denoted by this sense, the TR is occupying or using some parts of the LM. This phrasal verb is similar to the Extended Use / Occupy Sense of take. The meaning of up participating in this sense adds the More sense, as well as the Completion Sense, which seems to add emphasis. Most of the sentences in the corpus in which take up occurred have a negative connotation that is not found in sentences using simply the EU/Occupy Sense of take. The following sentences demonstrate this sense of the phrasal verb observed in the corpus:

(36) The rider was judged for taking up 2 seats.

(37) While it is entirely appropriate to see a doctor or nurse for a cough that has lasted three weeks or longer, seeing a GP or nurse for a niggling cough that has lasted a few days simply takes up everyone's time.

In this construction, $u p$ seems to be emphasizing the TR's act of occupying more than the norm or more than is necessary. Prototypically we expect $u p$ to have a positive connotation, but in this construction the $u p$ seems to highlight the notion that 'too much of a good thing' is negative.

In (36), the person is occupying two seats in the bus, one for sitting and the other for their bags, apparently when there were not enough seats for all the passengers. In (37), patients with minor sicknesses, including a trivial coughing condition, are using the practitioner's time when it is not warranted; 
in other words these patients are unnecessarily using the doctor's time that could be better spent on other patients with more serious illnesses.

4.3. OUT

This section presents the semantics of out and the phrasal verbs produced from the combination of get and take with this particle.

\subsubsection{Tyler and Evans' analysis of out}

According to Tyler and Evans (2003), in and out fall under a category of spatial particles that are sensitive to certain dimensions of the LM; in this case, the LM involves a bounded area. Bounded LMs are conceptualized as three -dimensional objects which possess an interior, a boundary, and an exterior. Human interaction with these objects has functional consequences, the most prominent one being the notion of containment. Containment itself involves a number of consequences. Bounded LMs constrain the movement of their TRs, as liquid in a bottle. In the case of a water bottle, the water is restricted to the shape and space of the interior of the bottle in which it is located. In certain cases, delimiting the movement of the TR can give rise to the notion of support, such as a cup containing a straw helping support the straw, allowing it to remain upright. The quality of the LM (opaque or clear) can also affect the specific construal of the scene, with opaque LMs blocking the view of the interior region. Thus, different aspects of our experience with containers can be coded in spatial particles in and out. The central meaning of out designates a relation in which the TR is exterior to a bounded LM. The LM is covert, which means it is not profiled by out. While the functional element (or the interactive relation between the TR and LM) associated with in is 'containment', out denotes the functional element of 'non-containment'. The central sense for out is illustrated in Figure 6.

The various senses of out are classified under clusters of meaning derived from the central sense, reflecting the various configurational and functional elements associated with bounded LMs. Table 1 illustrates the cluster of senses and the distinct senses proposed for out which were used in the analysis of the phrasal verbs in this study. The examples are from Tyler and Evans (2003).

The following section presents the analysis for the different verb-particle combinations with out. For each phrasal verb, a central sense and a number of distinct meanings are identified. The analysis involves a detailed explanation of each sense, followed by a number of examples from the corpus.

\subsubsection{Analysis of get out}

Central sense of get out. The phrasal verb get out in its central sense denotes a spatial meaning of an entity moving from an interior region to an exterior 


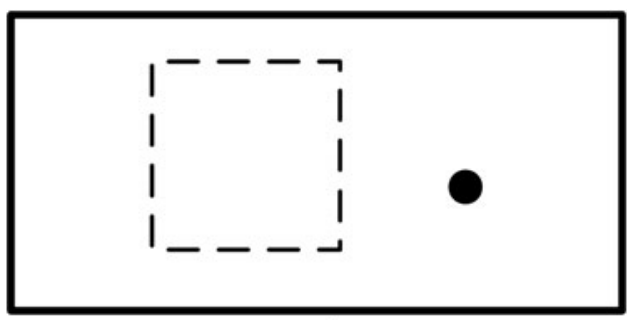

out

Fig. 6. Central sense of out. The shaded sphere represents the TR and the dashed square represents the covert bounded LM (Tyler \& Evans, 2003, p. 137).

region of a container. The location the entity is moving out of is conceptualized as a bounded LM and is often unspecified or covert and filled in by inference. In this use, the meaning of get is the extended Move Sense. The meaning of out is the central sense of designating 'a relation in which the TR is exterior to the bounded LM'. Figure 7 shows the central sense of get out.

Some examples of the central sense of get out are provided below:

(38) Ethan gets out, moves into the driveway, lines up his camera and snaps a photo.

(39) I was standing in the hallway while my boyfriend was getting the keys out .

In (38), the TR or the actor is moving from the interior space of the bounded LM, the car, to the exterior space. In (39), the person is causing the keys (TR) to move out of the bounded LM (probably his pants pocket); the LM is unspecified in this particular sentence and filled in by the interlocutors' background knowledge.

Distinct senses of get out. Three distinct senses of the phrasal verb were identified. These are explained below with examples for each sense.

Not in situ. This sense is closely related to the central sense. The key difference is that the $\mathrm{LM} /$ container is the home or typical place in which the TR is located. Additionally, the LM is usually not overtly mentioned. The construction means not being in that typical place. This sense draws on the extended Move Sense for get and the extended Not In Situ Sense for out. The following are some example sentences:

(40) You should get out more. It's not good to sit at home alone day after day.

(41) Come on. Let's get out and see a movie. 
T ABLE 1. Tyler and Evans' (2003) analysis of out

\begin{tabular}{lc}
\hline Clusters & \multicolumn{1}{c}{ Senses } \\
\hline The Location Cluster & 1-Not In Situ Sense: The TR is not in its default \\
& location where it spends extended periods of \\
time; e.g., She's out (for lunch) vs. She's in. & 2-No More Sense: There is no longer any of TR \\
& in the container so there is no more of the TR \\
available; e.g., We're out of milk. & 3- The Visibility Sense: The TR is located exterior \\
The Vantage Point Exterior Cluster & exterior to the bounded LM so the TR becomes \\
& visible; e.g., The moon is out. \\
4- The Knowing Sense: The TR is visible and as & a consequence it becomes known in certain \\
construals; e.g., They figured out the truth.
\end{tabular}

The data also contained a special, contextualized version of this sense in sentences such as the following:

(42) He recommends George H. W. Bush's courtesy, Bill Clinton's total recall of names and faces, and Barack Obama's focus on getting out the youth vote.

(43) Unlike Coakley, she'll share the ballot with Obama and other Democrats, meaning that the party's local machines will have no choice but to get out the vote - something they were accused of slacking on last time around.

The interpretation is that action was taken to encourage voters (TRs) to leave their typical locations (their places of work or their homes) for the purposes of voting. This contextualized variant always occurs with lexical phrase the vote, which is a metonym for the people who vote. The phrase the vote provides specific information about the purpose for moving the TRs to the exterior of the LM. Interpretation also involves our background knowledge concerning US elections.

Become known. This sense denotes that the TR is becoming known to the viewer. A consequence of the TR moving out of the bounded LM is that it often becomes visible or perceptually accessible to the viewer whose vantage point is exterior to the LM. According to Tyler and Evans (2003), the regular occurrence of a TR which was previously located inside the bounded LM and not being visible, subsequently becoming located exterior to the LM, has given rise to out developing an extended Visibility Sense. Once an object is visually accessible, it can be examined and hence 


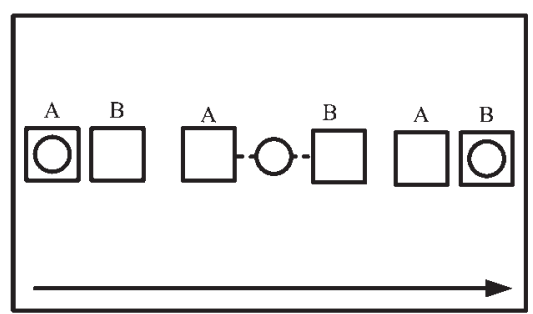

get

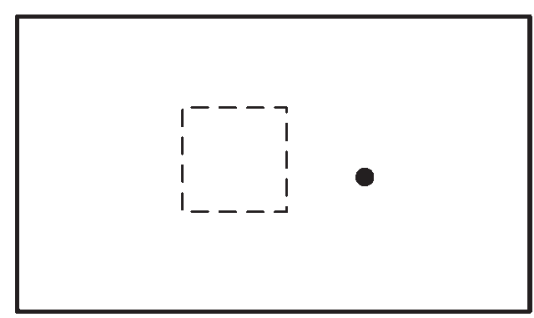

out

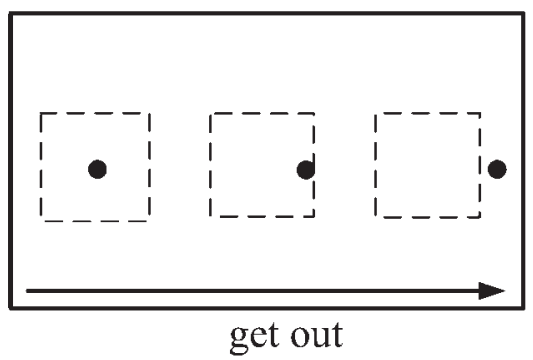

Fig. 7. The central sense of get out. The TR is represented by a dark sphere and the LM is represented by a dashed square. The time element is shown by an arrow and the bolded objects are profiled in the scene.

information can be gathered about it, i.e., it can become known. Indeed, one of the most prevalent metaphors in English is KNOWING IS SEEING, as expressed in sentences such as, I see what you mean, which is generally interpreted as 'I understand you' or I saw him for what he really was, which is generally interpreted as 'I knew his true character'. Through pragmatic strengthening, the correlation between seeing a previously contained entity and achieving a state of knowing about that entity has given rise to an additional, distinct sense of out, which is the Knowing Sense. Similarly, the phrasal verb get out has developed the distinct sense of Become Known. The meaning of get participating in this sense is the extended Change of State, with the TR going from a state of unknown to known. The following examples illustrate this sense of get out:

(44) My story had gotten out and suddenly I became the expert among my friends.

(45) I don't know how the secret got out.

In sentence (44), the speaker's story went from a state of being unknown to the friends, to the state of being known. In (45), the secret goes from a state of being unknown to a certain set of people, to being known. 
Become available. This sense denotes that the TR has become available. The sense of get participating in this meaning is again the extended Change of State Sense, specifically moving from an inaccessible to an accessible state; the sense of out participating is what we call the Accessibility Sense. Tyler and Evans (2003) do not identify this sense as a distinct sense for out. However, the analysis shows that there seems to be a sense of out which is related to the notion of accessibility. This sense is related to the Visibility Sense discussed above and can be explained in terms of our experience with containers. One frequently occurring consequence of being exterior to the LM (container) is that the TR or object inside the LM is inaccessible due to the features of the LM. This is especially true when the LM is closed on all sides. Once the TR is out of the container it is no longer confined by the LM and as a result it can become available and accessible to the experiencer. For instance, if jewels are locked in a safe, they are inaccessible (at least to those who do not know the combination for the safe) and not available to be worn. In contrast, if the jewels are not locked in the safe, if they are out on the dressing table, they are accessible and available to be worn. In this way out has come to develop an extended Accessibility Sense. In the following examples, the TR (invitation, report) have become available to the users:

(46) I finally got my invitations out for the wedding.

(47) The company got the report out on time.

In (46), the invitations go from a state of being inaccessible to the people being invited, to being accessible. In (47) the report (and the information contained in it) went from a state of being inaccessible to the public, to being accessible.

\subsubsection{Analysis of take out}

Central sense of take out. The central sense of take out denotes a spatial scene of a human hand removing an entity from a container. In many instances the LM is covert or unarticulated. The meaning of take in this construction is the central meaning of Get Hold Of and Remove, and the meaning of out is the central meaning of designating 'a relation in which the TR is exterior to a bounded LM'. Figure 8 represents the central sense of take out. The meaning of take is shown by the image of a hand getting hold of an object and subsequently moving the object from its original location; the meaning of out is the central sense of the TR located exterior to the LM.

Note that the TR is initially located in the interior of the bounded LM; in the final stage of the process the hand has moved the TR such that it is located exterior to the bounded LM. 
The following examples demonstrate the central sense of the phrasal verb:

(48) He took out a notepad and wrote a few sentences.

(49) I can't turn this off without taking out the battery.

Distinct senses of take out. Three distinct senses of the phrasal verb were identified. Below different senses of take out are explained and several examples are provided for each meaning.

Obtain a legal arrangement. The spatial configuration denoted by this scene is similar to the central sense in that the actor is removing an object (TR) from the container or the bounded LM. In this conceptualization the LM is an institution such as a bank or court from which a legal arrangement can be obtained. Historically, our recurrent experience with banks usually involves monetary transactions in which the person can physically obtain some money (this is still our experience with ATMs). Due to the high frequency of this type of experience with institutions such as banks, this sense has become conventionalized and take out has acquired this highly entrenched meaning of obtaining a legal (usually financial) arrangement. The following examples illustrate this sense of the phrasal verb:

(50) The notion of taking out a bank loan left a bad taste in his mouth.

(51) I encountered one family that had to take out a second mortgage on their home to get their child treatment.

This sense is also used in non-legal situations, as when someone with criminal intent takes out a contract on someone, meaning 'hires someone to kill a specified person'. We did not find examples of this use in the corpus, but novels and movies about organized crime are rife with them. We hypothesize that the use of the word contract, whose origins are in the legal system, has contributed to the expanded use of this sense.

Destroy an entity. This sense denotes that an entity is terminated or destroyed by another entity. The meaning of take in this use is the central Get Hold Of and Remove Sense. The verb designates the sense of getting control over the TR and exerting force on the TR. The meaning of out is the extended No More Sense. According to Tyler and Evans (2003), many of our experiences with containers involve a consumable entity such as food or beverage, which are by default located inside the region of the container. When the TR is consumed it is no longer located in the LM, and is, in fact, no longer in existence. An experiential correlation between a TR being exterior to the LM and the consequence of the TR no longer being available or no 


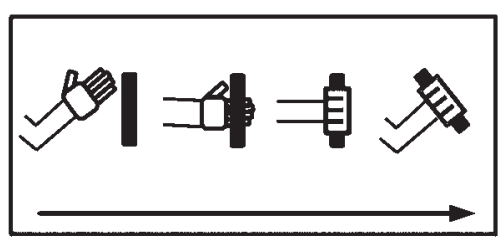

take

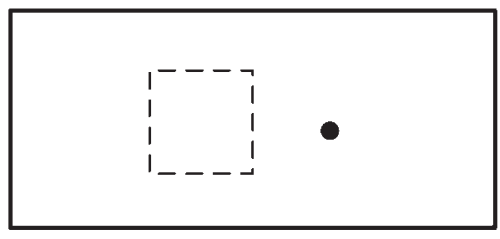

out

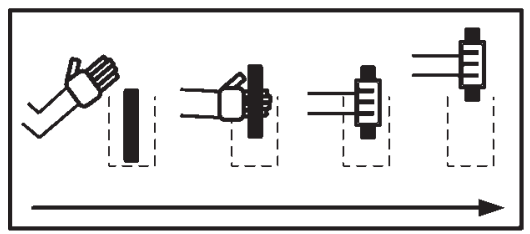

take out

Fig. 8. The central sense of take out. The time element is shown by an arrow and the bolded objects are profiled in the scene. In take up, the TR is represented by a bolded rectangle and the LM is represented by a dashed square.

longer existing has given rise to the No More Sense of out. The following examples demonstrate this sense of take out:

(52) John and Sam were still shooting as they took out soldiers, one by one.

(53) Last night four buildings were taken out by fire in my neighborhood.

In both these sentences, the entity being acted upon is destroyed.

Having a date. In this use, take out denotes the meaning of accompanying someone to an establishment or activity outside the home. The meaning of the verb participating is the central Get Hold Of and Remove Sense, which, recall, includes the meaning of moving the TR from its original location. The meaning of the particle is the extended Not In Situ Sense. As previously mentioned, in certain construals of out, the TR is no longer in its default location, such as home, in which it is normally located. Due to this conceptualization, out has developed the sense of 'exterior to the base location or home'. Many times we arrange a meeting with someone in a location outside the home, such as a restaurant or a bar. Often these meetings involve one person taking an active, agentive role and inviting the other to join in the activity. Having the agent in the active role of instigating the movement of the TR from an original location to a second location is consistent with the central use of take; the second location is designed by the extended sense of out. Due to the frequency of occurrence of this use in our daily experience, take out has acquired the meaning of having a date with a person. The following examples illustrate this sense of the phrasal verb: 
(54) For my 17th birthday, Harry took me out for ribs at the Singapor.

(55) He always likes to take her out to a very nice restaurant.

Note that the basic activity in both these sentences could be articulated simply with the verb take. Our hypothesis is that out provides an emphasis on moving from the TR's home, which can give rise to the inference of the activity having a special significance, which would be attached to a date.

\section{Conclusion}

In this study, we have examined the internal compositional interactions within a set of frequent and highly polysemous English phrasal verb constructions found in a set of naturally occurring data. Our basic hypothesis was that the seemingly intractable meanings of phrasal verbs can be systematically represented by taking into account the multiple meanings of both the particles and the verbs that make up the phrasal verb constructions. Tyler and Evans' (2003) Principled Polysemy Model was used to analyze the semantics of the particles and, in conjunction with Langacker's (1987, 1991) analysis, to offer an analysis of the semantics of the verbs in this study. Analyzing multiple meanings of the phrasal verbs provided strong evidence for the compositional nature of phrasal verbs, showing that the multiple meanings of each phrasal verb construction found in our data can be accounted for through the interaction of a particular, independently established, sense of the verb with a particular, independently established, sense of the particle. We found that a range of senses of the verb, including but not limited to the central sense, can combine with a range of senses of a particle. The result is that a phrasal verb construction, such as get out, can have several systematic, motivated meanings. Previous studies only considered the polysemy of the particle, and thus assumed a single, stable meaning for the verb participating in the construction. This approach repeatedly led to the conclusion that the meanings of the phrasal verb constructions were largely arbitrary. Our analysis has demonstrated that failure to consider the polysemy of both the verb and the particle and how the multiple meanings of each component contribute to the multiple meanings associated with the construction can only provide a partial analysis.

Additionally, rather than being fully compositional, the meaning networks of these phrasal verb constructions, like the meaning networks of prepositions and verbs themselves, is largely based on our conceptualization and background knowledge of the world. The notion of embodiment and humans' multiple experiences with and ability to shift construal on the same basic spatial scene is crucial for accounting for the range of different meanings of a phrasal verb 
construction. A wide range of phrasal verb meanings can be systematically motivated from the broad repository of human experiences.

The corpus data used for identifying the senses included 200 instances of each phrasal verb. Without investigating a larger corpus and consulting more resources, no claim can be made that all the distinct senses of each phrasal verb have been accounted for. In addition, while independent raters were used to code the meanings, due to the nature of categorization, the model proposed in this study and similar studies is partly subjective, and thus more research needs to be carried out in this area in order minimize possible researcher bias.

Future research can expand on the analysis of phrasal verbs by investigating other aspects of their meanings. One potential direction is to investigate the constraints on why particular senses of the verb and particle combine in certain uses. In general, while some senses of a specific verb and a specific particle do combine with each other to create a phrasal verb construction, other senses of the verb and particle do not combine. Motivation for these patterns of combination deserves further examination. In addition, the systematic nature of phrasal verbs, which traditional approaches had labeled as largely arbitrary, may have significance for other complex structures whose meaning is similarly considered opaque, such as many idiomatic phrases. Finally, the study has potential implications for teaching phrasal verbs to second language learners, as previous accounts have not provided a systematic, comprehensible rationale for learners. A meaningful representation of the polysemy networks of phrasal verbs could be utilized to help learners better understand the systematicity of the various senses of these complex lexical units.

\section{RE F E R E N C E S}

Bolinger, D. (1971). The phrasal verb in English. Cambridge, MA: Harvard University Press.

Brugman, C. (1981). Story of 'over'. (Unpublished MA thesis) University of California, Berkeley. Reprinted (1988) as The story of 'over': polysemy, semantics and the structure of the lexicon. New York: Garland.

Brugman, C., \& Lakoff, G. (1988). Cognitive typology and lexical networks. In S. Small, G. Cottrell, \& M. T. Tanenhaus (Eds.), Lexical ambiguity resolution (pp. 477-507). Palo Alto, CA: Morgan Kaufman.

Cann, R. (1993) Formal semantics: an introduction. Cambridge: Cambridge University Press.

Celce-Murcia, M., \& Larsen-Freeman, D. (1999). The grammar book: an ESL/EFL teacher's course (2nd edition). USA: Heinle \& Heinle Publishers.

Deane, P. (2005). Multimodal spatial representations: on the semantic unity of over. In B. Hampe (Ed.), From perception or meaning: image schemas in cognitive linguistics (pp. 235-282). Berlin: Mouton de Gruyter.

Dirven, R. (2001). English phrasal verbs: theory and didactic application, In M. Pütz \& S. Niemeier (Eds.), Applied cognitive linguistics, vol. II: language pedagogy (pp. 3-28). Berlin / New York: Mouton de Gruyter.

Evans, V. (2006). Lexical concepts, cognitive models and meaning-construction. Cognitive Linguistics, 17(4), 491-534. 


\section{MAHPEYKAR AND TYLER}

Evans, V. (2009). How words mean. Oxford: Oxford University Press.

Evans, V., \& Green, M. (2006). Cognitive linguistics: an introduction. Edinburgh: Edinburgh University Press.

Fraser, B. (1976). The verb-particle combination in English. New York: Academic Press.

Gamut, L. T. F. (1991). Logic, language and meaning, vol. 1: introduction to logic. Chicago: University of Chicago Press.

Goldberg, A. (1995). Constructions: a construction grammar approach to argument structure. Chicago: University of Chicago Press.

Grady, J. (1997). Foundations of meaning:primary metaphors and primary scenes. (Unpublished PhD dissertation) Department of Linguistics, University of California, Berkeley.

Grady, J. (1999). A typology of motivation for conceptual metaphor: correlation versus resemblance. In R. Gibbs \& G. Steen (Eds.), Metaphor in cognitive linguistics (pp. 79-100). Philadelphia: John Benjamins.

Hopper, P. J., \& Traugott, E. C. (1993). Grammaticalization (2nd ed.). Cambridge: Cambridge University Press.

Johnson, M. (1987). The body in mind: the bodily basis of meaning, imagination, and reason. Chicago/London: University of Chicago Press.

Johnson, C. (1999). Metaphor vs. conflation in the acquisition of polysemy: the case of see. In M. Hiraga, , C. Sinha, \& S. Wilcox (Eds.), Cultural, typological and psychological perspectives in cognitive linguistics (pp. 155-169). Amsterdam: John Benjamins.

Kennedy, A. (1920). The modern English verb-adverb combination (Language and Literature, I: 1). Stanford: Stanford University Press.

Lakoff, G. (1987). Women, fire and dangerous things: what categories reveal about the mind. Chicago/London: University of Chicago Press.

Lakoff, G., \& Johnson, M. (1980). Metaphors we live by. Chicago/London: University of Chicago Press.

Langacker, R. (1987). Foundations of cognitive grammar, vol. 1: theoretical prerequisites. Stanford: Stanford University Press.

Langacker, R. (1991). Foundations of cognitive grammar, vol. 2: descriptive application. Stanford: Stanford University Press.

Langacker, R. (2008). Cognitive grammar: a basic introduction. Oxford / New York: Oxford University Press.

Lindner, S. (1981). A lexico-semantic analysis of English verb-particle constructions with $u p$ and out. (Unpublished PhD dissertation) University of California, San Diego.

Lipka, L. (1972). Semantic structure and word-formation: verb-particle constructions in contemporary English. Munich: Wilhelm Fink Verlag.

Littlemore, J., \& Low, G. (2006). Figurative thinking and foreign language learning. Basingstoke: Palgrave Macmillan.

Live, A. (1965). The discontinuous verb in English. Word, 21, 428-451.

Mandler, J. (1992). How to build a baby: II. Conceptual primitives. Psychological Review, 99(4), 587-604.

Montague, R. (1974). Formal philosophy: selected papers of Richard Montague, edited and with introduction by Richmond H. Thomason. New Haven: Yale University Press.

Newman, J. (1996). Give: a cognitive linguistic study (Cognitive Linguistics Research 7). Berlin / New York: Mouton de Gruyter.

Quine, W. V. O (1970). Philosophy of logic. Englewood Cliffs: Prentice-Hall.

Rudzka-Ostyn, B. (2003). Word power: phrasal verbs and compounds (a cognitive approach). Berlin / New York: Mouton de Gruyter.

Sweetser, E. (1990). From etymology to pragmatics: metaphorical and cultural aspects of semantic structure. Cambridge: Cambridge University Press.

Talmy, L. (2000). Toward a cognitive semantics (2 vols.). Cambridge, MA: MIT Press.

Taylor, J. (2002). Cognitive grammar. Oxford: Oxford University Press.

Traugott, E. C. (1989) On the rise of epistemic meanings in English: an example of subjectification in semantic change. Language, 65(1), 31-55. 
A COGNITIVE LINGUISTICS ACCOUNT OF PHRASAL VERBS

Tyler, A. (2008). Applying cognitive linguistics to second language teaching: the English modals. In N. Ellis \& P. Robinson (Eds.), The handbook of cognitive linguistics and second language acquisition (pp. 456-488). Mahwah, NJ: Lawrence Erlbaum Associates.

Tyler, A. (2012). Cognitive linguistics and second language learning: theoretical basics and experimental evidence. New York, NY: Routledge.

Tyler, A., \& Evans, V. (2001). Reconsidering prepositional polysemy networks: the case of over. Language, 77(4), 724-765.

Tyler, A., \& Evans, V. (2003). The semantics of English prepositions: spatial scenes, embodied meaning and cognition. Cambridge: Cambridge University Press.

Vandeloise, C. (1991). Spatial prepositions: a case study in French. Chicago: University of Chicago Press. 